\title{
THE TRANSITION BETWEEN TRICKLE FLOW AND PULSE FLOW IN A COCURRENT GAS-LIQUID TRICKLE-BED REACTOR AT ELEVATED PRESSURES
}

\author{
W. J. A. WAMMES, S. J. MECHIELSEN and K. R. WESTERTERP ${ }^{\dagger}$ \\ Department of Chemical Engineering, Chemical Reaction Engineering Laboratories, University of Twente, \\ PO Box 217, 7500 AE Enschede, Netherlands
}

(First received 3 July 1989; accepted in revised form 2 January 1990)

\begin{abstract}
The effect of reactor pressure in the range of $0.2-2.0 \mathrm{MPa}$ on the transition between the trickle-flow and the pulse-flow regime has been investigated for the non-foaming water-nitrogen and aqueous $40 \%$ ethyleneglycol-nitrogen systems. Most models and flow charts which are all based on atmospheric experiments were able to describe the transition experiments performed at $0.2 \mathrm{MPa}$ reasonably well. However, this is not so at elevated pressures. For both gas-liquid systems it is found that at a constant superficial gas velocity and higher reactor pressures the transition of trickle flow to pulse flow occurs at a relative higher liquid throughput. In the pressure range of $2.5-7.0 \mathrm{MPa}$ the pulse-flow regime could not be obtained for throughputs up to $1.7 \mathrm{~cm} / \mathrm{s}$ superficial liquid velocity. An explanation for this effect is given based on the dynamic liquid hold-up and pressure drop data determined at the flow regime transition. Finally an empirical equation is proposed, relating the parameters governing the flow regime transition and describing the hydrodynamic conditions at the transition between trickle flow and pulse flow.
\end{abstract}

\section{INTRODUCTION}

The three-phase reactor in which a liquid phase and a gas phase flow concurrently downward through a fixed bed of catalyst particles is commonly called a trickle-bed reactor. The advantages and disadvantages of the trickle-bed reactor compared to other types of three-phase reactors are discussed by Satterfield (1975) and Tarhan (1983). It is used extensively in the chemical and petrochemical industry and, especially to improve the liquid solubility of the gaseous reactant, it is operated at elevated pressures in most of the applications.

In the trickle-bed reactor basically four flow regimes can be encountered, depending on the gas and liquid flow rate and the fluid and packing properties. At low liquid and gas flow rates the liquid trickles over the packing and the gas phase is the continuous phase filling the remaining voids. This regime is known as the trickle-flow regime. The pulsing regime can be obtained at higher liquid and gas loads and here the liquid periodically blocks the channels between the packing particles and forms plugs. Above this liquid plug the gas pressure increases to such values that the plug is subsequently blown down through the column. The spray flow regime occurs at relatively high gas rates and low liquid rates: the liquid phase is entrained in droplets by the gas phase. This regime is also called the mist flow regime. At low gas and high liquid flow rates the liquid becomes the continuous phase and the gas flows downward in the form of bubbles: this is the dispersed bubble flow regime. The transition between the pulse-flow and the trickle-flow regime is sharp, while the transition to spray flow and dispersed bubble flow is more gradual.

\footnotetext{
† Author to whom correspondence should be addressed.
}

Knowledge of the several transitions is indispensable in the design of trickle-bed reactors; several studies show that the pressure drop, liquid saturation, liquid mixing and heat and mass transfer coefficients are affected differently in each regime [see, for example, Specchia and Baldi (1977), Ruether et al. (1980) and Rao and Drinkenburg (1985)].

In the literature a number of studies have been presented on the transitions between the several regimes and their dependence on the gas and liquid flow rates, the liquid properties and the packing geometry and size. Regretfully - at least to our knowledge-no research has yet been carried out on the effect of the pressure of the gas phase. Hence there is still a lag between the published experimental research and industrial practice at elevated pressures.

In the present study the experimental determination of the transition of the trickle-flow to the pulseflow regime at varying reactor pressures and with two non-foaming Newtonian gas-liquid systems will be presented. To get a better understanding of the transition phenomenon we have also determined the dynamic liquid hold-up as well as the pressure drop at each point of transition. With these new available data we will test the different models, correlations and flow charts for the prediction of flow pattern boundaries. Based on our experimental results at elevated pressures we will give the hydrodynamic parameters which are in our view governing the transition between the trickle-flow and the pulse-flow regime. These hydrodynamic parameters will be related into an equation which describes the hydrodynamic condition at the initiation of the pulses in the column.

\section{LITERATURE SURVEY}

In this survey we mainly focus our attention on the transition between trickle flow and pulse flow for 
non-foaming gas-liquid systems. More extensive reviews on the several flow regime transitions are given by Gianetto et al. (1978) and Herskowitz and Smith (1983).

Extensive experimental data on the transition between the trickle-flow and the pulse-flow regime are available for atmospheric conditions. From most studies only qualitative conclusions can be drawn on the dependence of the flow regime transition on the gas and liquid velocities, the physical properties of the fluids and the packed-bed properties. In only a few cases has an attempt been made to correlate the data. Table 1 summarizes these studies.

Charpentier and Favier (1975) proposed a flow map based on an empirical coordinate system originally developed by Baker (1954) for gas-liquid flow regime transitions in empty horizontal pipes. The reactor pressure has been incorporated in the gas mass flux $G$. The parameters $\lambda$ and $\varphi$ are empirical dimensionless numbers which contain the physical properties of the gas-liquid system as related to the air-water system. The boundary line in the flow diagram separating the trickle-flow from the pulse-flow regime is based on atmospheric experiments and several gas-liquid systems. The gas density has been varied hetween 0.15 and $1.8 \mathrm{~kg} / \mathrm{m}^{3}$ by using helium and carbon dioxide gas.

The coordinate system of the flow map proposed by Talmor (1977) is based on a semi-theoretical approach. Which flow regime is encountered depends on the ratio of the superficial gas to liquid velocities and on the ratio of the inertia and gravity driving forces to the viscous and surface tension resisting forces. For the definitions of the dimensionless numbers in the $x$-coordinate we refer to Talmor (1977). For nonfoaming systems five different flow regimes are distinguished: the transitions are indicated by curves based on his own experimental data and those of several other authors. The line separating the trickle-flow regime from the pulse-flow regime is mainly based on data obtained with air and water-glycerol mixtures.

Fukushima and Kusaka (1977) investigated the transition between flow regimes in a column with 8-10 particles on a diameter. It is obvious that -if the dimensionless number $\left(D_{r} / d_{p}\right)^{0.5}$ in Table 1 plays a role-the contribution of the wall flow to the hydrodynamic behaviour was relatively large. No influence of $D_{r} / d_{p}$ on the hydrodynamics was found by Blok et al. (1983) and Sicardi et al. (1979). According to Herskowitz and Smith (1983) the effect of the wall flow can be disregarded at $D_{r} / d_{p}>18$.

Sicardi et al. (1979) and Sicardi and Hofmann (1980) considered the trickle bed as consisting of parallel channels with constrictions. They assumed that the pulse-flow regime occurs when large waves on the liquid surface occlude the constrictions. A dimensionless correlation was derived by which the ratio of the wave amplitude to the mean liquid film thickness $(A / \delta)_{\mathrm{tr}}$ is described as a function of the tangential stress on the liquid surface at the point of transition, the surface tension and the constriction geometry. The two constants in eq. (3) in Table 1 were determined by means of transition experiments with several types of packing.

Blok et al. (1983) have found experimentally the mean actual liquid velocity $u_{t}$ to be constant at each point of transition between trickle and pulse flow. Therefore they postulated that the initiation of pulses is determined by a critical minimum value of the Froude number, based on the mean actual velocity $u_{l}$ [see eqs (4) and (5) in Table 1]. For the air-water system and for several types of packing the critical value was experimentally found to be 0.09 .

The model of $\mathrm{Ng}(1986)$ is based on theoretical considerations about the hydrodynamics at the scale of a particle in the packed bed. A pulse is formed in the pore neck of the constriction between two particles due to the fusion of the two rippled liquid films on the particles. An expression is derived for the actual gas velocity in the pore neck, $u_{p n}$, just before the pulse formation. To calculate the value of the parameter $\alpha$, the fraction of the pore constriction occupied by the liquid, $\mathrm{Ng}$ (1986) used the liquid hold-up correlation of Wijffels (1974), which has been developed in the absence of gas flow. Therefore the value of $\beta_{\text {tot }}$ and hence the value of $\alpha$ will be overestimated. In this model the role of the gas density is explicitly taken into account by eq. (6) in Table 1 . However, this model does not consider that at the same time the total liquid hold-up and hence $\alpha$ in eq. (8) also depend on the gas density: an increase in $\rho_{g}$ will result in a higher pressure drop over the bed and therefore in a decrease in the liquid hold-up. It can be seen easily that the effects of the gas density on $u_{p n}$ and $\alpha$ affect $v_{g}$ in opposite ways [see eqs (7) and (8) in Table 1].

\section{EXPERIMENTAL INSTALLATION}

The major problem in the design of a gas-liquid installation suitable for a large range of pressures is the large variation of gas mass fluxes necessary to obtain sufficient high superficial gas velocities under each condition. We solved this problem by recycling the gas phase around the reactor.

A flow sheet of the experimental installation, which has been designed for hydrodynamic experiments up to pressures of $7.0 \mathrm{MPa}$, is given in Fig. 1. Two gas-liquid systems are used in this study: water-nitrogen and aqueous $40 \%$ ethyleneglycol-nitrogen. The physical properties of both liquids, the reactor dimensions and the operating limits are listed in Table 2.

\section{The trickle-bed reactor}

The trickle-bed reactor (4)- the numbers refer to Fig. 1-consists of three cylindrical tubes of $1 \mathrm{~m}$ length and an inner diameter of $D_{r}=51 \mathrm{~mm}$. The top and bottom sections have been made of 316 stainless steel tubes and the middle section is of a transparent polycarbonate material. The top and bottom sections are thermostatted at $293 \mathrm{~K}$ by heated water flowing through a coil.

The shape and the dimensions of the transparent section are outlined in Fig. 2. We have tested the tube 


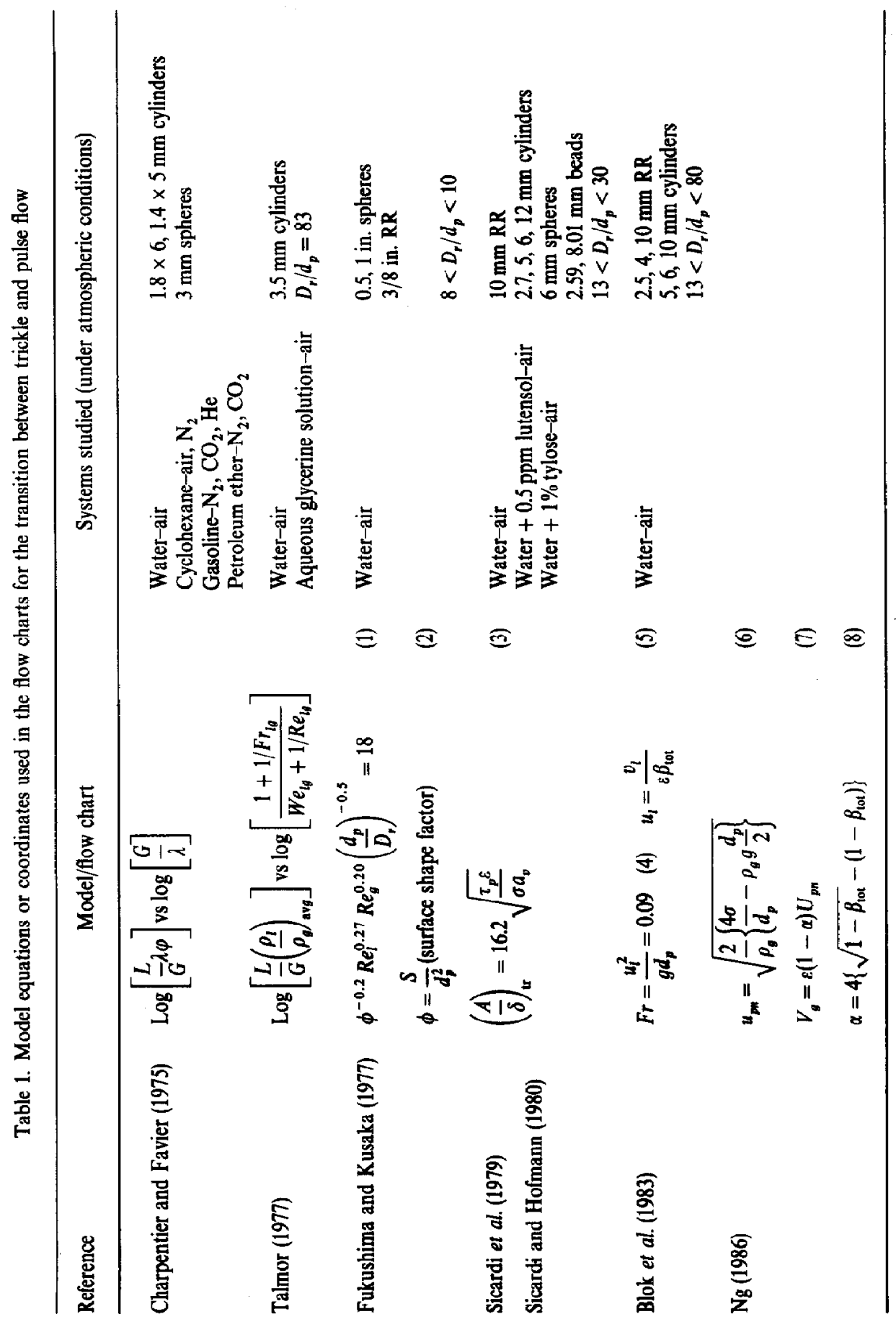




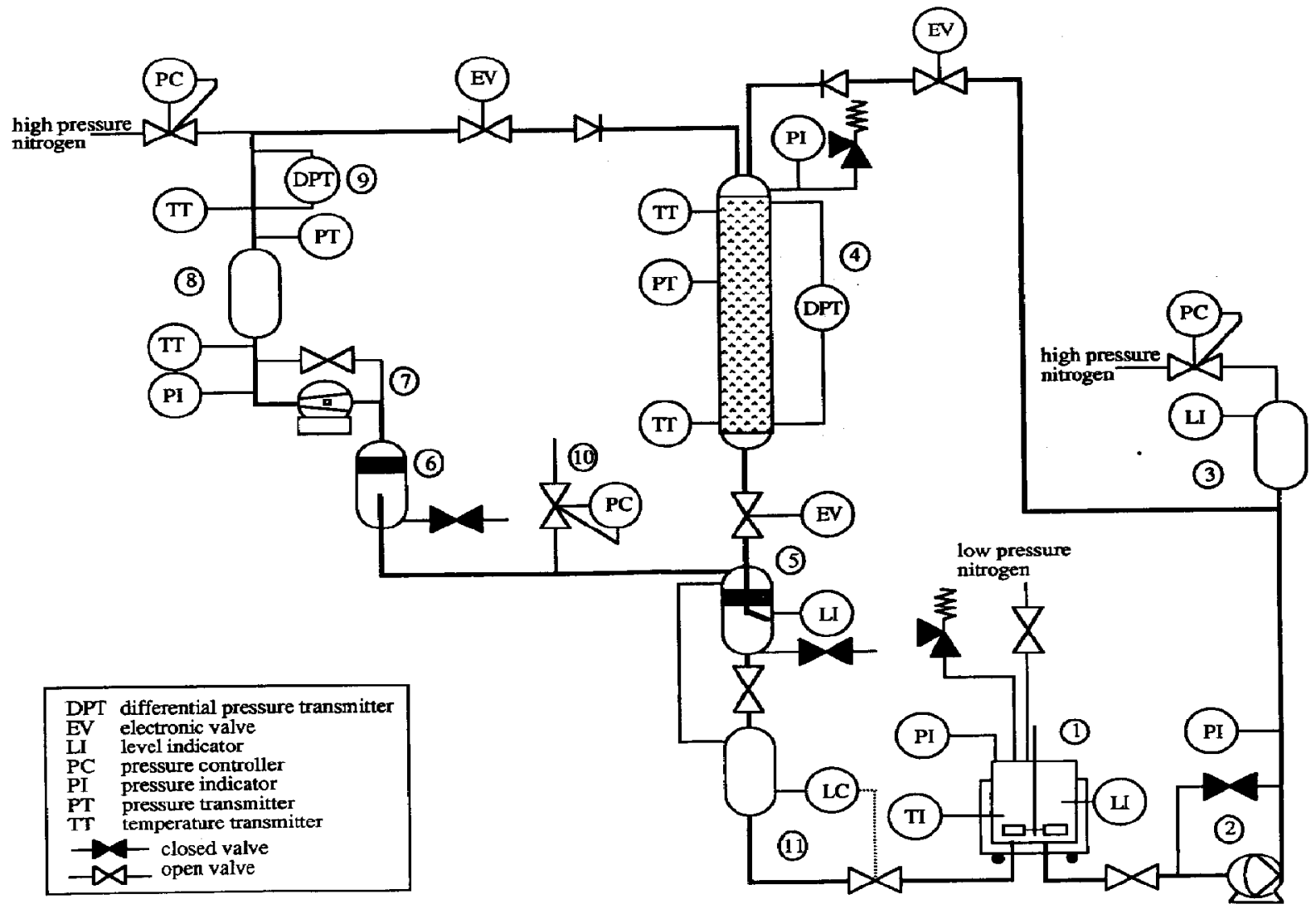

Fig. 1. Flow sheet: $1=$ liquid storage vessel, $2=$ liquid pump, 3 = air-chamber, $4=$ trickle-bed reactor, 5 = gas-liquid-separator, $6=$ demister, $7=$ gas booster, $8=$ gas buffers, $9=$ orifices, $10=$ system back pressure, 11 = level-controlled liquid buffer vessel.

Table 2. Reactor dimensions, operating limits and physical properties of the liquid phases.

\begin{tabular}{lccc}
\hline Reactor dimensions & $D_{r}=51 \mathrm{~mm}$ & $d_{p}=3 \mathrm{~mm}$ (glass spheres) & $\varepsilon=0.39$ \\
$L=2.62 \mathrm{~m}$ & $\frac{\rho_{l}\left(\mathrm{~kg} / \mathrm{m}^{3}\right)}{1000}$ & $\eta_{l}\left(\times 10^{3} \mathrm{Ns} / \mathrm{m}^{2}\right)$ & $\sigma_{l}\left(\times 10^{3} \mathrm{~N} / \mathrm{m}\right)$ \\
Physical properties & 1050 & 1.0 & 72 \\
Water & & 2.9 & 60 \\
$40 \%$ ethyleneglycol & & $v_{t}<31 \times 10^{-2} \mathrm{~m} / \mathrm{s}$ & $P_{r}<7.5 \mathrm{MPa}$ \\
Limit values in our installation & & & \\
$v_{l}<1.8 \times 10^{-2} \mathrm{~m} / \mathrm{s}$ & &
\end{tabular}

up to a pressure of $10 \mathrm{MPa}$ and measured the increase in length and outer diameter as a function of the pressure. The expansion of the material was found to be elastic in the applied pressure range. At $10 \mathrm{MPa}$ the increase in length, the smallest and largest outer diameter was, respectively, $1.2,0.5$ and $0.2 \mathrm{~mm}$. Because of the material expansion at elevated pressures the suspension of the tube has been made flexible.

Glass spheres of $d_{p}=3 \pm 0.5 \mathrm{~mm}$ are used as the packing material. Before loading the reactor the spheres are thoroughly cleaned with acetone. The support grid for the packing is located $8 \mathrm{~cm}$ above the bottom flange. A well-packed bed has been established by loading the reactor in batches of one-third of the total amount of packing material and vibrating the column after loading each batch until the height of the bed has become constant. The final height of the packed bed is $L=2.62 \mathrm{~m}$ and the bed has an overall porosity of $\varepsilon=0.39$.

A spray device with 40 holes of $0.75 \mathrm{~mm}$ is used to obtain a uniform distribution of the liquid at the top of the packed bed. The device is positioned at such a height above the bed that the liquid is evenly distributed without spraying it on the wall of the reactor. 


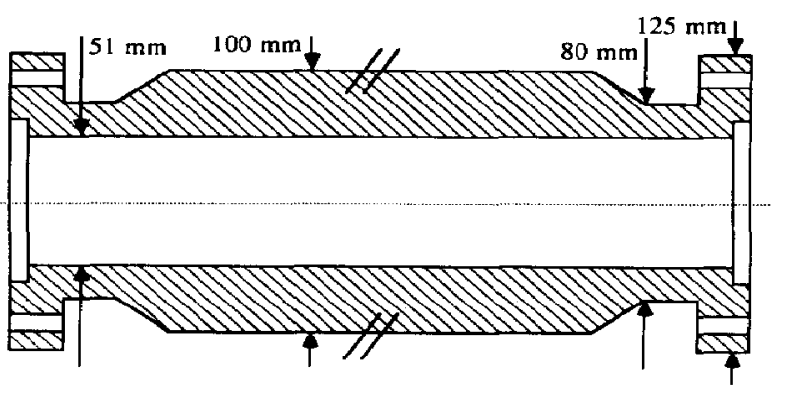

Fig. 2. Dimensions and shape of the polycarbonate tube.

A pressure transmitter and a thermocouple are connected to the reactor wall at a location near the top of the packed bed. At several positions on the wall of the reactor provision has been made to connect a differential pressure transmitter. The stability of the reactor operation can be observed accurately by a continuous recording of the pressure drop signal.

\section{The liquid circulation system}

The liquid is kept in a 601 storage vessel (1) which is thermostatted at $293 \mathrm{~K}$ and pressurized at $0.05 \mathrm{MPa}$ $\mathbf{N}_{2}$. From this vessel the liquid is pumped to the installation by means of a Lewa-membrane pump type EL6000. The pump (2) has a maximum capacity of $40 \mathrm{ml} / \mathrm{s}$ and the liquid flow is controlled by varying the stroke length of the piston. It can be adjusted either manually or electronically. For calibration two burettes, one for high and one for low liquid flow rates, are available. At the discharge side of the pump an air-chamber (3) with a volume of 51 is installed to prevent pulsations in the liquid flow. The liquid level can be regulated by means of a venting pressure regulator. After the liquid has passed the reactor it is separated from the gas phase and fed to a levelcontrolled buffer vessel (11), from which it is returned back to the low-pressure storage vessel (1).

\section{The gas circulation system}

The experimental installation is pressurized with nitrogen from the general-purpose nitrogen feed system of the laboratory which is maintained at 300 bar. The pressure in the trickle-bed installation is manually adjustable by a Tescom 1100 series pressure regulator and a Tescom 1700 series back pressure regulator (10).

The gas phase is circulated by means of a Haskel gas booster (7) type AGD-7 which is driven by compressed air and has a maximum volumetric capacity of 2.3 actual $\mathrm{m}^{3} / \mathrm{h}$. In order to separate entrained liquid droplets from the gas phase a metal demister sponge is installed in the gas-liquid-separator (5) and another one in a second demister vessel (6).

Additional buffer vessels (8) with a total volume of 101 have been installed in the discharge line of the gas booster to prevent pressure and flow fluctuations. Suction pressure fluctuations are damped in the gas-liquid-separation vessel and the demister vessel.
The gas flow is regulated manually by a needle valve in the recycle flow around the gas booster. The gas mass flow is measured by means of an orifice meter (9). Guidelines for the design of the orifice meter were taken from Perry and Green (1984). Two sharp edged orifice plates of $0.5 \mathrm{~mm}$ thickness with an orifice diameter of $\mathbf{4 m m}$ for the low gas mass flow rates and $7 \mathrm{~mm}$ for the high ones are installed in two parallel tubes. The gas pressure and the temperature are measured at the orifice inlet side. The pressure drop across the orifice is measured by a Honeywellsmart transmitter, type STD120. The superficial gas velocity in the reactor is calculated on the basis of the measured gas mass flow rates at the orifice together with the pressure and the temperature of the gas at the bed entrance.

\section{Automation}

The operation of the experimental installation is automated except for the pressurizing of the installation and the setting of the gas flow rate. A programmable Analog Devices $\mu$ mac- 5000 data acquisition and control unit connected to an Apple $2 \mathrm{C}$ micro computer is used for process control and monitoring. The liquid pump and the magnetic valves at the reactor inlet and outlet can be actuated by the computer. The stroke length of the piston can be adjusted automatically. The pressure, the pressure difference and the temperature both of the trickle-bed reactor and of the orifice meter are recorded continuously. These data together with the calculated superficial gas and liquid velocities in the reactor are monitored.

\section{EXPERIMENTAL PROCEDURES}

The transition between trickle flow and pulse flow is observed visually in the transparent middle section of the column. In the following we will use the concept of transition point in the sense of the combination of pressure and flow rates at which pulses are observed with a frequency between 0.3 and $0.5 \mathrm{~Hz}$. Several methods are used to find the transition point. At constant gas flow rate the liquid flow rate is changed or the gas flow rate is changed at constant liquid flow rate until the transition is observed, whereas the experiment is started from either the trickle-flow regime or the pulsing-flow regime. We noticed that after adjusting a new operating point it could take a few minutes before the trickle-bed installation operated steadily again. Therefore the signal of the reactor pressure drop is recorded continuously for at least 5 min after each change to check whether the behaviour has become steady.

The dynamic liquid hold-up is determined at each point of transition with the "stop flow" method. The magnetic valves in the gas and liquid inlet pipes and the reactor outlet are closed simultaneously by means of the computer. Then after emptying the gas-liquidseparator the valve between the gas-liquid-separator and the liquid buffer vessel is closed while the reactor outlet valve is opened. The liquid now drips out of the 
column into the separator. The column is empty of free liquid after a certain period. Subsequently the collected liquid is pressed out of the separator and weighed.

To determine the minimum drainage-time and the reproducibility of this method we performed a set of experiments in which the leak-out time was varied between $15 \mathrm{~min}$ and $\mathbf{4 8} \mathrm{h}$. For water and for the aqueous $40 \%$ ethyleneglycol solution a leak-out time of $30 \mathrm{~min}$ and $1 \mathrm{~h}$, respectively, is required to obtain the correct liquid hold-up values. The "stop flow" method gives reproducible hold-up data with a relative error of less than $5 \%$. The dimensionless dynamic liquid hold-up $\beta_{\mathrm{dyn}}$ is calculated as the ratio between the collected liquid volume and the free volume $c V$, of the packed bed.

\section{EXPERIMENTAL RESULTS AND DISCUSSION}

The transition between the trickle-flow regime and the pulse-flow regime is plotted in the Figs 3 and 4 as a function of the superficial gas velocity, the liquid velocity and the reactor pressure for the water-nitrogen and aqueous $40 \%$ ethyleneglycol-nitrogen systems.

It has been visually observed that for each reactor pressure the first appearance of the pulses is located in the lower part of the transparent column section. This has also been reported by other investigators [e.g. Tosun (1984) Talmor (1977)]. It is caused by the increase in the gas velocity in the flow direction as a consequence of the pressure gradient over the reactor. After another slight increase in the gas throughput the pulsations are observed over the entire length of the transparent section.

The transition lines are independent of the method used, whether - at a constant liquid velocity - the gas velocity is varied or the liquid velocity is varied at a constant gas velocity or whether the measurements are started in the trickle-flow regime or the pulse-flow regime. Hence we find the transition to be independent on any previous operating conditions in the column. This was also reported by Christensen et al. (1986) and Levec et al. (1988).

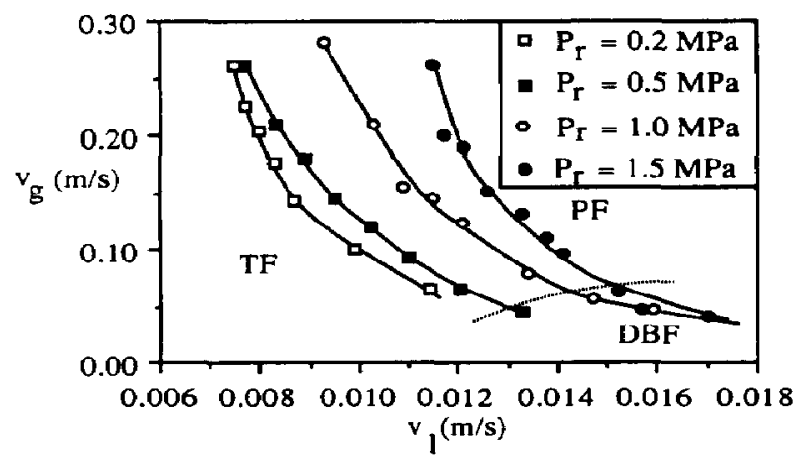

Fig. 3. Effect of the reactor pressure on the boundary lines between trickle and pulse flow for the water-nitrogen system: $\mathbf{T F}=$ trickle flow, $\mathbf{P F}=$ pulse flow, $\mathbf{D B F}=$ dispersed bubble flow.
In Fig. 3 for the water-nitrogen system we also marked the transition to the dispersed bubble flow regime. It must be considered to be only indicative because we could not define a sharp point of transition. In this transitional range the liquid has a slowly pulsating behaviour and by increasing the liquid velocity the gas phase becomes gradually more dispersed in the form of bubbles.

Figures 3 and 4 show that the transition depends strongly on the pressure in the reactor. Though the shape of the curve is only slightly altered with varying pressure, there is-at a constant superficial gas velocity-a shift towards higher liquid velocities with an increase in the reactor pressure. With the water-nitrogen system we operated the reactor at $2.5,4.0$ and 7.0 $\mathrm{MPa}$ with different gas to liquid velocity ratios but we could not detect visually the pulse-flow regime anymore, at least within our operating limits.

A comparison of the transition lines for both systems at $1.0 \mathrm{MPa}$ shows that for aqueous $40 \%$ ethyleneglycol-nitrogen the transition between trickle flow and pulse flow occurs at relatively lower superficial liquid velocities. This has also been reported for atmospheric experiments with the aqueous $20 / 40 \%$ glycerol-air system by Sai and Varma (1988).

\section{Comparison with the literature}

From the power law relation of eq. (1) as derived by Fukushima and Kusaka (1977) one can conclude that, if we introduce our experimental data in this relation, the equation does not describe the pressure influence on the transition between trickle flow and pulse flow: an increase in the reactor pressure and therefore also in $R e_{g}$ would lead to a lower value of $R e_{t}$ at the transition, which is not in agreement with our observation of an increase in the superficial liquid velocity to obtain the pulse-flow regime at higher pressures (see Figs 3 and 4 ).

To test the theoretical model of $\mathrm{Ng}$ (1986), we first compared the liquid hold-up correlation used by $\mathrm{Ng}$ for the calculation of the fraction of the pore constriction occupied by the liquid, $\alpha$, with our experimental liquid hold-up data. In all cases the correlation over-

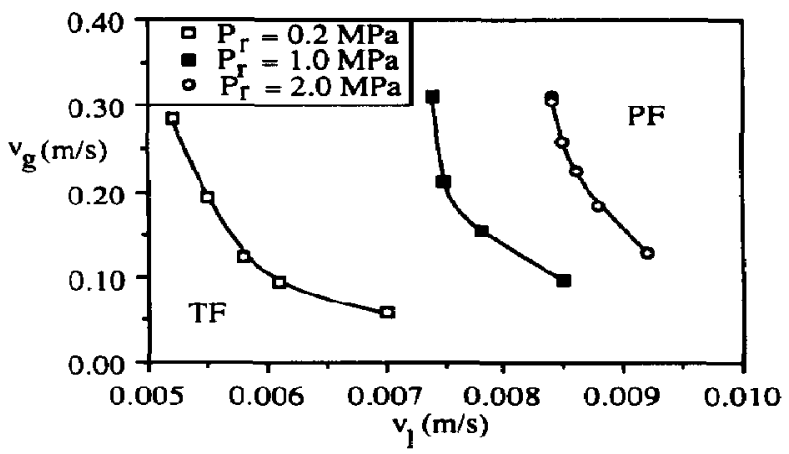

Fig. 4. Effect of the reactor pressure on the boundary lines between trickle and pulse flow for the aqueous $40 \%$ ethyleneglycol-nitrogen system. 
estimated the liquid hold-up, probably because the reactor pressure drop-one of the driving forces for the liquid phase flow-is not taken into account in the hold-up equation. Therefore our own experimental liquid hold-up data have been used to calculate the value of $\alpha$. By substituting the hold-up and density values, belonging to each experimental point of transition, into the model eqs (6) and (8) the predicted value of $v_{g}$ is calculated. A comparison of the predietcd $v_{g}$ values with the experimental data shows that the predictions are a factor 3-10 too high.

The transition data of both gas-liquid systems at a reactor pressure of $0.2 \mathrm{MPa}$ are reasonably well described by the flow diagram of Charpentier and Favier (1975) and the equation of Blok et al. (1983). However, that is not so at higher reactor pressures. In the diagram of Charpentier and Favier (1975) the transition line at elevated pressures shifts towards higher values of $G / \lambda$. With respect to the model of Blok et al. (1983) the Froude number on the transition line at an elevated pressure is not constant anymore but increases with $v_{g}$. This becomes more pronounced at higher pressures.

The model equation of Sicardi et al. (1979) and Sicardi and Hofmann (1980) [see eq. (3)] can be rearranged to eq. (9) [see Sicardi et al. (1986)], which relates the pressure gradient to the total liquid holdup at the transition:

$$
\beta_{\mathrm{trt}, \mathrm{1 \textrm {r }}}=0.066\left(\frac{\sigma_{1} a_{v}^{2}}{\varepsilon^{2}}\right)^{0.45}\left(\frac{\Delta P}{L}\right)_{\mathrm{tr}}^{-0.45}
$$

To calculate the total liquid hold-up we estimated the static liquid hold-up at 0.11 for both liquids by means of the diagram given by Charpentier et al. (1968). Equation (9) describes our transition data determined at $0.2 \mathrm{MPa}$ well. However, at higher pressures for a given hold-up value a higher pressure drop or higher tangential stress is necessary to initiatc pulses.

The flow diagram developed by Talmor (1977) gives a good prediction for the transition of the trickle-flow regime to the pulse-flow regime, when applied to the

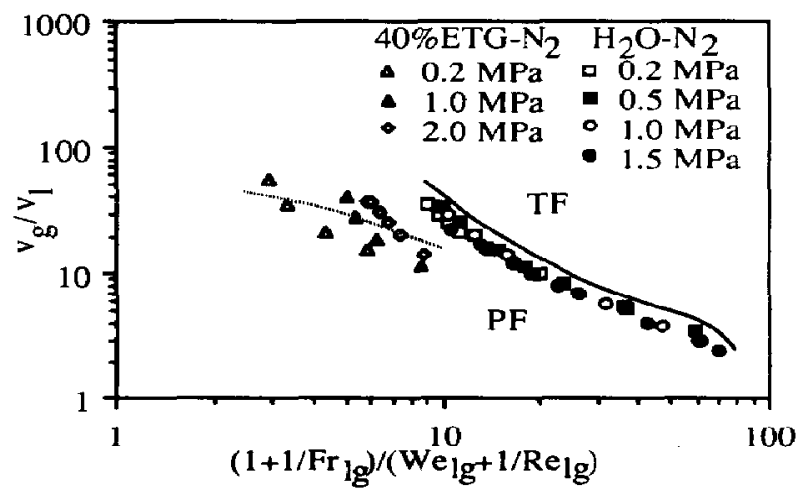

Fig. 5. Transition points of both gas-liquid systems plotted in the Talmor diagram: $(\ldots .$.$) transition between trickle$ flow and foaming pulse flow, (- - ) transition between trickle flow and pulse flow for non-foaming systems. water-nitrogen system at elevated pressures (see Fig. 5). Our data are positioned in the pulse-flow area just below the proposed boundary line. This is probably due to a slightly different definition of the point of transition. Our data obtained with the aqueous $40 \%$ ethyleneglycol-nitrogen system do not coincide with the boundary line, despite the fact that this line is also based on experiments with aqueous glycerol solutions.

The pulses observed with the aqucous $40 \%$ ethyleneglycol solution may be considered as slightly foaming or cloudy pulses so we have also marked in Fig. 5 the boundary line for foaming systems as proposed by Talmor (1977). In this case a better agreement is obtained between our data and the proposed transition between the trickle-flow and the foamingpulse flow regime. Unfortunately there is still no precise definition nor a universal measurement method to characterize foaminess: therefore we make the comparison with some reservation.

From these comparisons it can be concluded that our data obtained at a reactor pressure of $0.2 \mathrm{MPa}$ fit reasonably well into most of the proposed models and flow charts. However, it is obvious that our data at elevated pressures do not fit into the model equations or flow charts, except in the flow diagram proposed by Talmor (1977) and then only for the water-nitrogen data.

The reactor pressure drop and the dynamic liquid holdup at the flow regime transition

In our view the main effect of a pressure elevation, at a constant superficial gas and liquid velocity, is the influence of the increased pressure gradient over the packed bed on the hydrodynamic state. The reactor pressure drop and the gravitational force are the driving forces acting upon the liquid phase. For various reactor pressures the relative contribution of the pressure gradient to the total driving force for liquid flow at the flow regime boundary is plotted in Fig. 6 as a function of the superficial gas velocity for the water-nitrogen system. At these operating conditions the contribution of the pressure drop is large and becomes more pronounced at elevated pressures due

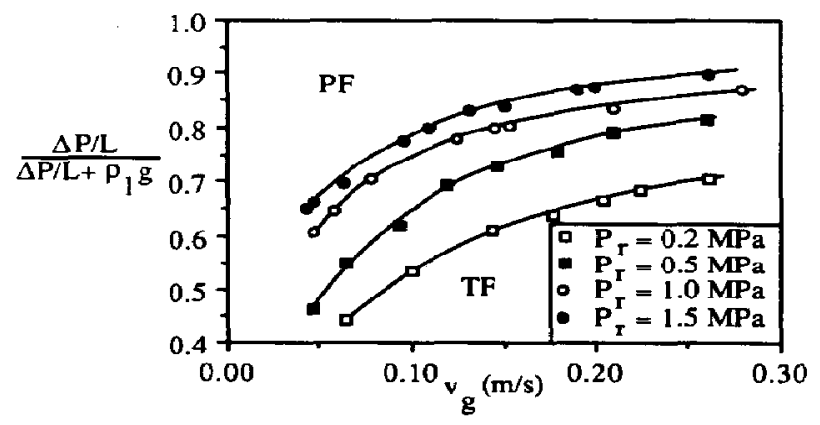

Fig. 6. Relative contribution of the pressure drop to the total driving force acting upon the liquid phase at the transition points of the water-nitrogen system. 
to the higher density of the gas phase. The $40 \%$ ethyleneglycol-nitrogen system follows a similar trend. Hence the liquid hold-up is noticeably influenced by the value of the pressure gradient under the operating conditions near the flow regime boundary. Generally speaking, a pressure elevation at constant superficial gas and liquid velocities will always lower the liquid hold-up because of the increased pressure gradient at higher gas densities. The influcnce of a reactor pressurc variation on the liquid hold-up gives us a qualitative explanation for the observed shift in Figs 3 and 4 of the flow regime boundary towards higher $v_{l}$ at elevated pressures. In Figs 7 and 8 the dynamic liquid hold-up $\beta_{\text {dyn,tr }}$ at the transition for various reactor pressures is plotted as a function of the superficial gas velocity. Figures 7 and 8 show that at a constant gas velocity $v_{g}$ and higher reactor pressure only a slightly lower liquid hold-up is required for the transition from trickle flow to pulse flow, and at relatively higher gas velocities a lower liquid hold-up is necessary to initiate the pulses. When the trickle-bed reactor operates just in the pulse-flow regime and only the reactor pressure is set to a higher value, the liquid hold-up will decrease as a consequence of the increase in the pressure gradient over the bed. After the reactor is operating steadily again the liquid hold-up at the higher reactor pressure will have a value below the $\beta_{\mathrm{dyn}, \mathrm{tr}}$ corresponding to the setpoint of $v_{g}$ and $P_{r}$ (see Fig. 7 or 8). In our opinion now the mean thickness $\delta$ of the liquid film on the packing particles, which is proportional to the total liquid hold-up according to eq. (10) and in the order of $0.1 \mathrm{~mm}$, is too thin to collapse in the constrictions between the particles, so no pulses can be formed any more:

$$
\delta \approx \frac{\varepsilon \beta_{\text {tot }}}{a_{v}}=\frac{\varepsilon\left(\beta_{\mathrm{dyn}}+\beta_{\mathrm{stat}}\right)}{a_{v}}
$$

in which $\beta_{\text {stat }}$ has a constant value.

Thus as the result of the reactor pressure increase the hydrodynamic regime shifts from pulse-flow to trickle-flow because the liquid hold-up becomes too low. If subsequently-at constant gas velocity and reactor pressure-the liquid velocity $v_{l}$ is increased, the liquid hold-up and the film thickness will grow

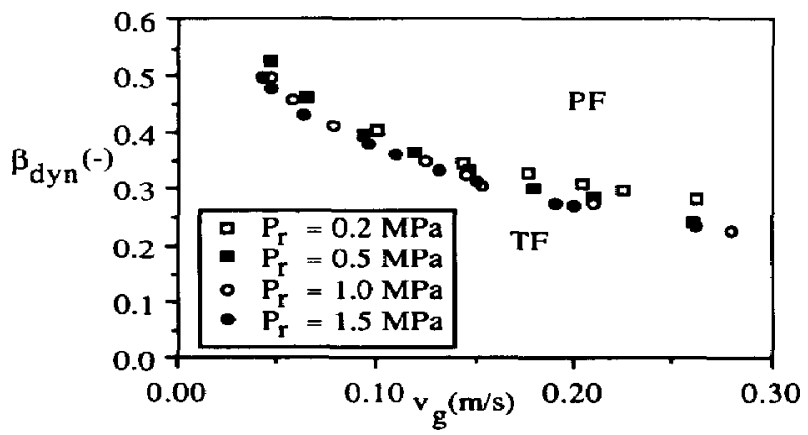

Fig. 7. Dynamic liquid hold-up at the transition points for the water-nitrogen system. again. When the liquid hold-up reaches the critical film thickness corresponding to the setpoint of $v_{g}$ and $P_{r}$, the hydrodynamic regime will shift from trickleflow to pulse-flow again. Therefore at higher reactor pressures the liquid throughput must be increased to give a sufficiently high liquid hold-up to initiate the pulse-flow regime. The film thickness at the pulse initiation for aqueous $40 \%$ ethyleneglycol is higher than for water.

To develop a model which describes the flow regime transition on a theoretical basis seems at this stage to be very difficult because the understanding of the origin of the pulses is still very limited. Many operating variables play a role: the superficial gas and liquid velocity, the liquid viscosity and surface tension, the particle geometry and the porosity of the packed bed [see, for example, Sato et al. (1973), Chou et al. (1977) and Sai and Varma (1988)]. Furthermore from our own experimental research we concluded that the transition is also influenced by the pressure in the trickle-bed reactor. To derive a condition which describes the hydrodynamic state at the start of the pulse-flow regime we followed an empirical approach. The critical film thickness above which the films collapse and subsequently block the constrictions is not a constant value but depends on the size of the disturbances at the film surface, as was stressed by Sicardi et al. (1979) [see eq. (9)]. These disturbances are influenced by the liquid properties, the gas velocity and the gas density as can be concluded from Figs 7 and 8. For given liquid and packed-bed properties the condition at the transition can be described as a first approximation by

$$
\beta_{\text {tot, tr }}=c_{1} v_{g}^{c_{2}} \rho_{g}^{c_{3}}
$$

and for

$\beta_{\text {tot }}<\beta_{\text {tot tr: }}$ : operation in the trickle-flow regime $\beta_{\text {tot }}>\beta_{\text {tot. tr }}$ : operation in the pulse-flow regime.

In order to calculate $c_{1}, c_{2}$ and $c_{3}$ for both gas-liquid systems we minimized the sum of the squared residuals. The result is

$$
\beta_{\mathrm{tot}, \mathrm{tr}}=c_{1} v_{g}^{-0.26} \rho_{g}^{-0.04}
$$

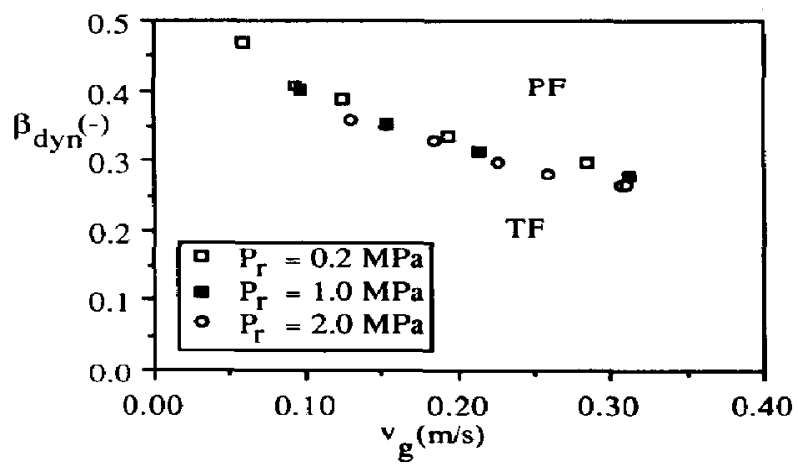

Fig. 8. Dynamic liquid hold-up at the transition points for the aqueous $40 \%$ ethyleneglycol-nitrogen system. 
with

$c_{1}=0.27$ for water-nitrogen

$c_{1}=0.32$ for aqueous $40 \%$ ethyleneglycol-nitrogen

and a mean relative deviation of, respectively, 3 and $4 \%$.

It must be stressed that additional experiments in the trickle-flow and the pulse-flow regime must be performed to check whether the above-mentioned condition can be applied and that transition experiments have to be performed with gases of different molecular weight. Moreover, to be able to present the condition in a dimensionless form the liquid and bed properties must be varied.

\section{APPLICATION TO COMMERCIAL TRICKLE-BED REACTORS}

An industrial trickle-bed reactor generally is operated at elevated pressures. From our work with nitrogen gas we have seen that at relatively high reactor pressures the pulse-flow regime cannot be attained any more at realistic liquid flow rates: due to the high pressure drop the liquid hold-up always remains below its critical value. In the case of high-pressure processes with non-foaming systems and with a gas phase of a molar weight comparable to or higher than nitrogen, e.g. in the case of oxidation processes, it will probably be impossible to operate the reactor in the pulse-flow regime. The situation will be different for hydrogenation processes where the pressure drop will be much lower because of the lower gas density at equal operating pressure and temperature. Hence we think that for hydrogen gas the pulse-flow regime might occur at realistic flow rates even at high pressures of say $10 \mathrm{MPa}$.

In the foregoing we have presented results and conclusions on the basis of experimental research in a small-diameter column. Although the wall effects are likely to be negligible on the overall hydrodynamic behaviour some remarks must be made when applying data from small-scale laboratory columns to the large industrial trickle-bed reactors. In the small-scale columns it is observed that at the onset of the pulse-flow regime the pulses span the entire column diameter. Christensen et al. (1986) investigated the hydrodynamic behaviour in a trickle-bed reactor with a rectangular cross-sectional area of $0.46 \times 0.05 \mathrm{~m}^{2}$. They reported that at the flow regime transition the pulses did not span the entire crosssection despite the good gas-liquid distribution at the top of the bed: considerably higher flow rates were necessary to obtain pulses spanning the entire crosssection. In the case of very large column diameters it might be that only local pulses occurs. The operating conditions at the onset of the local pulses were found to be comparable to the flow regime transition conditions in small-diameter columns. Sicardi et al. (1979) experimented with columns up to $0.3 \mathrm{~m}$ in diameter but the existence of local pulses was not mentioned by them.
Furthermore two major differences must be remarked-besides the likely existense of local pulse formation-when applying the results from laboratory scale to a commercial trickle-bed reactor. First of all in the large industrial columns it is difficult to obtain a uniform packed bed when loading of the catalyst. Second, in the commercial reactors the reactions are mostly exothermic. Because of the low capability to remove the heat of reaction the trickle-bed reactors are operated more or less adiabatically in contrast to the isothermal conditions under which the hydrodynamic behaviour is studied in the laboratory. Hence the physical properties of the gas-liquid system in the axial direction can vary greatly if there is a temperature gradient over the column. These two differences lead to varying hydrodynamic states in the axial as well as the radial direction throughout the reactor.

Despite the differences in the experimental conditions between laboratory and industrial practise we are convinced that the results obtained from smalldiameter columns are very helpful in predicting the flow regime in a large industrial trickle-bed reactor taking the aforementioned differences into account.

\section{CONCLUSIONS}

Up till now experimental research of the transition between the several hydrodynamic regimes in the concurrent gas-liquid trickle-bed reactors has been restricted to atmospheric conditions. This is in contrast to industrial practice where a vast majority of the trickle-bed processes are operated at elevated pressures. In our study the transition between the trickleflow regime and the pulse-flow regime has been investigated with water-nitrogen and aqueous $40 \%$ ethyleneglycol-nitrogen systems at elevated pressures up to $7.0 \mathrm{MPa}$ and by means of visual observation. For this purpose a reactor section of $1 \mathrm{~m}$ length has been constructed of transparent polycarbonate material and tested up to $10 \mathrm{MPa}$. From measurements with both gas-liquid systems it was found that the flow regime boundary shifts towards higher superficial liquid velocities when the reactor pressure is increased. This effect was explained as follows: an increase in the reactor pressure-at constant superficial gas and liquid velocities - results in a higher pressure drop over the packed bed due to the higher density of the gas phase. The pressure drop is a driving force acting on the liquid phase, besides the gravitational force, and thus decreases the dynamic liquid hold-up. The mean liquid film thickness, which is proportional to the hold-up, becomes smaller and subsequently the liquid films cannot collapse any more to initiate the pulses. Therefore at an elevated pressure a higher liquid throughput is necessary to obtain a sufficiently high liquid hold-up for pulse formation. In the pressure range of 2.5-7.0 MPa the pulse-flow regime could not be detected visually for the throughputs up to $1.7 \mathrm{~cm} / \mathrm{s}$ superficial liquid velocity.

From the different flow charts and equations proposed in the literature only the flow diagram of 
Talmor (1977) gave a good prediction for the transition at elevated pressures, regretfully only for the water-nitrogen system.

When applying the information on the flow regime transition based on research performed in small-scale laboratory set-ups to the industrial practice several differences must be regarded. At this moment it is not possible to give accurate predictions of the flow regime boundary in commercial reactors because of the lack of information on the additional hydrodynamic effects on a large scale, such as local pulsing. However, the aforementioned results on the pressure effect might be helpful in estimating the flow. regime boundary in the large-scale reactors operating at elevated pressures.

Acknowledgements - The investigations were financially supported by the Netherlands Foundation for Chemical Research (STW) and DSM Research BV. We also acknowledge A. Pleiter and his assistants for their technical support.

\section{NOTATION}

\begin{tabular}{|c|c|}
\hline$a_{v}$ & $\begin{array}{l}\text { specific packing surface area, } \mathrm{m}^{2} / \mathrm{m}^{3} \text { of } \\
\text { packed bed }\end{array}$ \\
\hline $\boldsymbol{A}$ & wave amplitude, $\mathrm{m}$ \\
\hline$c$ & constant \\
\hline$d_{p}$ & equivalent particle diameter, $\mathrm{m}$ \\
\hline$D_{r}$ & reactor diameter, m \\
\hline Fr & Froude number $\left[u_{l}^{2} /\left(g d_{p}\right)\right]$ \\
\hline $\boldsymbol{g}$ & gravitational acceleration, $\mathrm{m} / \mathrm{s}^{2}$ \\
\hline$G$ & gas mass flux, $\mathrm{kg} /\left(\mathrm{m}^{2} \mathrm{~s}\right)$ \\
\hline$L$ & liquid mass flux, $\mathrm{kg} /\left(\mathrm{m}^{2} \mathrm{~s}\right)$ \\
\hline$L_{r}$ & reactor length, m \\
\hline $\begin{array}{l}P_{r} \\
\Delta P / L\end{array}$ & $\begin{array}{l}\text { reactor pressure, } \mathbf{M P a} \\
\text { pressure gradient, } \mathbf{N} / \mathrm{m}^{3}\end{array}$ \\
\hline $\operatorname{Re}$ & Reynolds number $\left(\rho v d_{p} / \eta\right)$ \\
\hline$S$ & geometric packing area, $\mathrm{m}^{2}$ \\
\hline $\boldsymbol{u}$ & ictual or interstitial velocity, $\mathrm{m} / \mathrm{s}$ \\
\hline$v$ & $\begin{array}{l}\text { superficial velocity based on empty cross- } \\
\text { section, } \mathrm{m} / \mathrm{s}\end{array}$ \\
\hline$V_{r}$ & reactor volume, $\mathbf{m}^{3}$ \\
\hline
\end{tabular}

\section{Greek letters}

$\alpha \quad$ fraction of the pore constriction occupied by the liquid phase

$\beta_{\text {dyn }} \quad$ dynamic liquid hold-up, $\mathrm{m}^{3} / \mathrm{m}^{3}$ reactor void volume

$\beta_{\text {tot }}$ total liquid hold-up, $\mathrm{m}^{3} / \mathrm{m}^{3}$ reactor void volume

$\delta \quad$ mean liquid film thickness, $m$

$\varepsilon \quad$ porosity of the packed bed

$\eta \quad$ dynamic viscosity, $\mathrm{N} s / \mathrm{m}^{2}$

$\rho$ density, $\mathrm{kg} / \mathrm{m}^{3}$

$\sigma \quad$ surface tension, $N / m$

$\tau$ tangential stress at the gas-liquid interface, $\mathrm{N} / \mathrm{m}^{2}$

\section{Subscripts}

$g$ referring to the gas phase

$l \quad$ referring to the liquid phase

pn referring to the pore neck between particles tr referring to the transition between pulse and trickle flow

\section{REFERENCES}

Baker, O., 1954, Simultaneous flow of oil and gas. Oil Gas $J$. 53, $185-191$.

Blok, J. R., Varkevisser, J. and Drinkenburg, A. A. H., 1983, Transition to pulsing flow, hold-up and pressure drop in packed columns with cocurrent gas-liquid downflow. Chem. Engng Sci. 38, 687-699.

Charpentier, J. C. and Favier, M., 1975, Some liquid hold-up experimental data in trickle-bed reactors for foaming and non-foaming hydrocarbons. A,I.Ch.E. J. 21, 1213-1218.

Charpentier, J. C., Prost, C., van Swaaij, W. P. M. and LeGoff, P., 1968, Etude de la rétention de liquide dans une colonne à garnissage arrosé à co-courant et à contrecourant de gaz-liquide. Génie chim., Paris 99, 803-826.

Christensen, G., McGovern, S. J. and Sundaresan, S., 1986, Cocurrent downflow of air and water in a two-dimensional packed column A.I.Ch.E. J. 32, 1677-1689.

Fukushima, S. and Kusaka, K., 1977, Interfacial area and boundary of hydrodynamic flow region in packed column with cocurrent downward flow. J. chem. Engng Japan 10, $461-467$.

Gianetto, A., Baldi, G., Specchia, V. and Sicardi, S., 1979, Hydrodynamics and liquid solid contacting effectiveness in trickle-bed reactors. A.I.Ch.E. J. 24, 1087-1104.

Herskowitz, M. and Smith, J. M., 1983, Trickle-bed reactors: a review. A.I.Ch.E. J. 29, 1-19.

Levec, J., Grosser, K. and Carbonell, R. G., 1988, The hysteretic behaviour of pressure drop and liquid hold-up in trickle-beds. A.I.Ch.E. J. 34, $1027-1030$.

$\mathrm{Ng}, \mathrm{K}$. M., 1986, A model for flow regime transitions in cocurrent downflow trickle-bed reactors A.I.Ch.E. J. 32, 115-122.

Perry, R. H. and Green, D., 1984, Perry's Chemical Engineers' Handbook, 6th Edition, pp. 5.14-5.16. McGraw-Hill, New York.

Rao, V. G. and Drinkenburg, A. A. H., 1985, A model for pressure drop in two-phase gas-liquid downflow through packed columns. A.I.Ch.E. J. 31, 1010-1018.

Ruether, J. A., Yang, C. and Hayduk, W., 1980, Particle mass transfer during cocurrent downward gas-liquid flow in packed beds. Ind. Engng Chem. Process Des. Dev. 19, 103-107.

Sai, P. S. T. and Varma, Y. B. G., 1988, Flow pattern of the phases and liquid saturation in gas-liquid cocurrent downflow through packed beds. Can. J. chem. Engng 66, 353-360.

Sato, Y., Hirose, T., Takahashi, F., Toda, $M$. and Hashiguchi, Y., 1973, Flow pattern and pulsation properties of cocurrent gas-liquid downflow in packed beds. J. Chem. Engng Japan 6, 315-319.

Satterfield, C. N., 1975, Trickle-bed reactors. A.I.Ch.E. J. 21 , $209-228$.

Sicardi, S., Gerhard, H. and Hofmann, H., 1979, Flow regime transition in trickle-bed reactors. Chem. Engng J. 18, 173-182.

Sicardi, S., Gerhard, M. and Hofmann, H., 1984, Some observations concerning pressure drop-hold up correlations in trickle-bed reactors. Chem. Engng Commun. 42, 1-15.

Sicardi, S. and Hofmann, H., 1980, Influence of gas velocity and packing geometry on pulsing inception in trickle-bed reactors. Chem. Engng J. 20, 251-253.

Specchia, V. and Baldi, G., 1977, Pressure drop and liquid hold-up for two-phase cocurrent flow in packed beds. Chem. Engng Sci. 32, 515-523.

Talmor, E., 1977, Two phase downflow through catalyst beds. A.I.Ch.E. J. 23, 868-874.

Tarhan, M. O., 1983, Catalytic Reactor Design, pp. 155-160. McGraw-Hill, New York.

Tosun, G., 1984, A study of cocurrent downflow of nonfoaming gas-liquid systems in a packed bed. Ind. Engng Chem. Process Des. Dev. 23, 29-35.

Wijffels, J. B., Verloop, J. and Zuiderweg, F. J., 1974, Wetting of catalyst particles under trickle-flow conditions. Chem. Reaction Engng II, Adv. Chem. Ser. 133, 151-163. 